\title{
AUTHOR AND SUBJECT INDEX
}

abbreviations 2

Abraham, H.J. 98

acknowledgments 8

Alley, C.O. 86

astrolabe 4, 10, 13, 25, 63, 77, 105

Bender, P.L. 86

BIH 2, 5f, 37, 63, 77

Bomford, G. 3, 37

chains of stations $3,10,25$

concurrent observations 25

continental drift $3,10,13,19,25,33,44 f, 52$, 57,80

- estimates 19

corner-reflector 86

crustal displacements, see continental drift

earth tides 71

Feissel, M. (Miss) 63

Fujii, S. 45

Garland, G.D. 19

geodetic problems 37

Gougenheim, A. 95

Guinot, B. 3f, 63

introduction 2

ILS 2f, 5, 13, 25, 33, 44, 52, 77, 82, 84, 101

IPMS 2, 5f, 12, 70

laser 86

latitude, secular variation $3,30,33,44,52,80$, 90

Local Committee $7 f$

longitude, secular variation $3,31,45,57,90$

Markowitz, W. 9, 25

mean pole of $1903.05 f, 13,30,37$

Melchior, P. 71

moon 86

'new system, 1900-05', see mean pole of 1903.0

Nicolini, T. 101
Okazaki, S. 45

Okuda, T. 44

organization of Symposium 7

origin for polar motion, see mean pole of 1903.0

paleomagnetism $3,19,80$

Participants 15

polar motion $25,37,52,63,77,80,90,95,98$, 101

pole, origin, see mean pole of 1903.0

- secular motion 3, 25, 33, 80, 98

PZT 2, 4, 10, 13, 30, 63

radio tracking 91

Rapid Latitude Service 5

reflecting astrolabe 105

resolutions 6,13

Robbins, A.R. 37

rotation of earth $31,71,80,98$

Runcorn, S.K. 80

satellite techniques $4,7,14,86,91$

Scientific Councils 6

secular motions, see latitude, longitude, and pole

Staff 16

Stoyko, A. (Mme) 52

Stoyko, N. 57

Sugawa, C. 44

Takagi, S. 77

Thomas, D. V. 105

time and polar motion 63,77

Torao, M. 45

Trask, D.W. 91

Vegos, C.J. 91

Vicente, R.O. 100

Wakō, Y. 33

Working Group 4, 10

Yumi, S. 33 